\title{
Effect of Nursing Care Standards for Preventing Deep Vein Thrombosis among Patients Undergoing Hip Surgery on Nurses' Performance and Patients' Outcome
}

\author{
Doaa M. El-SayedEad ${ }^{1}$, KameliaF. Abd-Allah $^{2}$, Mohamed O. Hegazi ${ }^{3}$, Sabah S. \\ Mohammed $^{4}$ \\ ${ }^{1}$ clinicalinstructor at Benha technical health institute, \\ ${ }^{2}$ Professor of Med-Surgical Nursing, Faculty of Nursing, Ain shams Univeristy. \\ ${ }^{3}$ Professor and head of orthopedic department Faculty of medicine Benha University \\ ${ }^{4}$ Lecturer of Medical surgical nursing, Faculty of Nursing, Benha University.
}

E-mail sabah.abdlrazek@fnur.bu.edu.eg

\begin{abstract}
:
Aim of this study wasto evaluate the effect of Nursing care standards (NCSs) for preventing deep vein thrombosis(DVT) among patients undergoing hip surgery on nurses' performance and patients' outcome. Research hypothesis are (1): Orthopaedic nurses' performance level for prevention DVT will be improved post NCSs implementation than pre implementation. (2): The incidence of DVT among patients undergoing HS post NCSs implementation (study group) will be lesser than pre implementation (control group).

Research design: A quasi-experimentalwas utilized.

Setting:The study was carried out at orthopaedic unit and outpatient at Benha university hospital.

Subjects: 30nurses who working in orthopaedic unit.Purposive sample of 132 patients were divided into equally study and control groups.

Tools: Data were collected through four main tools: nurses' interview questionnaire sheet,nurses' practice observational checklist, hospital structure checklist andpatient's outcome assessment sheet.

Results: 70\%\&80\% of nurses had unsatisfactory level of knowledge and practice scores preimplementation.Meanwhile, 96.7\%\&93.3\% of nurses had satisfactory level of knowledge and practice scorespost implementation respectively. The incidence of DVT among patients in study group was lesser than patients in control group with highly statistically significant improvement in patients' outcome $(P \leq 0.001)$.

Conclusion: there was statistically significant improvement in nurses' performance and reducing incidence of DVT among the patients in study group than the patients in control group.

Recommendation:Orientation programs should be provided for all newly nurses about the developed NCSs for preventing DVT among patients undergoing $H S$.
\end{abstract}

Keywords:Deep vein thrombosis, Hip surgery, Nursing care standards,Patients' outcome, Performance

\section{Introduction}

Hip surgery (HS) including total hip arthroplasty (THA) and hip fracture surgery (HFS) are the major orthopedic surgical procedures which can cause high morbidity and even mortality. Venous thromboembolism (VTE) is one of the most serious complications associated with HS, and it comprises deep vein thrombosis (DVT) and pulmonary embolism (PE).TheVTE is a major life threating complication in patients undergoing HS and a major cause of death during the first three months after surgery ${ }^{[1]}$. The DVT is a major medical problem characterized by thrombi formation in the deep venous system and can result in a fatal PE. This problem occurs most commonly in the legs, although thrombi can also form in the veins of the arms as well ${ }^{[2]}$.

The incidence of asymptomatic DVT after a major orthopedic surgery without prophylaxis reportedly ranges from $30 \%$ to $80 \%$ whereas the incidence of symptomatic DVT reportedly ranges from $0.5 \%$ to $4 \%$. About $40-50 \%$ of untreated symptomatic DVT patients will develop a PE within 3 months and 10\% of symptomatic PE cases will die within 1 hour of onset ${ }^{[3]}$.In Egypt, a study conducted at El-Hadara orthopedic and traumatology university hospital in Alexandria in 2005 for development of an assessment scale for patients at risk of DVT reported 14\% incidence of DVT among lower limb orthopedic patients after one month follow up period post discharge ${ }^{[4]}$.

The DVT is a common and potentially lethal disease which leads to severe morbidity with poor quality of life and even sudden death related to PE. Prevention is more important and cost effective than treatment, because once DVT develops; it can only be cured at considerable expense. Since the diagnosis is difficult and also the treatment is not always satisfactory, the prevention of DVT is obviously essential. Prevention of DVT 
is the effective approach to reduce death from PE and morbidity from DVT. Prevention decreases length of hospital stay, improves quality of life, decreases rehabilitation time and decreases economic burden ${ }^{[5]}$.

Orthopedic nurses play an important role in the detection, treatment and prevention of DVT among patients undergoing HS because those patients are needed for nursing interventions for DVT prevention as integral part of nursing care standards (NCSs). An awareness of all aspects of DVT is vital in providing optimal nursing care for patients undergoing HS in order to improve patients' outcome, reduce the incidence and potentially life threatening complications of DVT ${ }^{[6]}$.

A standard is defined as a performance model which is based on a desired level of excellence. The standards of professional nursing practice coincide with the steps of the nursing process to represent the directive nature of the standards as the professional nurse completes each component of the nursing process which include assessment, diagnosis, expected outcome, implementation and evaluation. The NCSs can be classified according to hospital structure, nursing process and patients' outcome. The Standards have been written so as to be easily accessible to a variety of groups, including nurse practitioners, governments, regulatory agencies, health care professionals and the community ${ }^{[7]}$.

\section{Significance of the study}

Orthopedic nurses can play a major role in DVT prevention if well-educated and empowered to change hospital culture. Their increased level of knowledge undoubtedly leads to an improvement in the delivery of patient care. Appropriately trained nurses are skilled in assessing the risk of DVT in patients with HS and ensuring prophylactic measures are in place for those patients who are particularly vulnerable to developing DVT. Nurses are well positioned to improve outcome of patients with HS. Nurses should be involved in establishing protocols for DVT prevention and in ensuring their implementation. The DVT prevention should be incorporated into NCSs that are useful for improving the quality of patient'scare ${ }^{[8]}$.It is obvious that there is a felt need to establish such a standard with the ultimate goal of prevention and optimal health for patientswith $\mathrm{HS}^{[4]}$. However it is not only the need to establish NCSs for preventing DVT among patients with HS but also there is a felt need to implement and evaluate the effect of these standards on nurses' performance and patients' outcome.

\section{Aim Of The Study}

The present study aimed to evaluate the effect of nursing care standards for preventing deep vein thrombosis among patients undergoing hip surgery on nurses' performance and patients' outcome

\section{Research hypothesis:}

- Orthopedic nurses' performance level (knowledge and practice) for prevention DVT will be improved post NCSs implementation than pre implementation.

- The incidence of DVT among patients undergoing HS post NCSs implementation (study group) will be lesser than pre implementation (control group).

\section{Subjects and methods:}

Research Design:A quasi-experimental research design was utilized to conduct the aim of this study.

Setting:This study was conducted atorthopedic unit and outpatient orthopedic clinic at Benha university hospital.

Subjects: Two groups of subjects were included in the study:

(1) All available nurses $(\mathbf{n}=\mathbf{3 0})$ who have been working in orthopedic unit at Benha university hospital, and agree to participate in the study.

(2) A purposive sample of all adult patients from both gender who were undergoing HS, their age ranged between $18>60$ years old and have no DVT on admission to orthopedic unit at Benha university hospital, and willing to participate in the study (n=132). Which were divided into (66) patients (control group) pre implementing NCSs and (66) patients (study group) post implementing NCSs.

\section{Tools of data collection:}

First tool: interview questionnaire sheet for nurses:

It was constructed by the researcher after reviewing relevant literature. It was written in simple Arabic language for assessing nurses' knowledge regarding NCSs for preventing DVT among patients undergoing HS (pre \& post NCSs implementation). It included three parts:

Part (A): Demographic characteristics of nurses; such as age, gender, level of education, years of experiences and previous attendance of training courses related to preventing DVT among patients undergoing HS.

Part (B): Nurses' knowledge about DVTwhich included 23 items about hip surgery complications, characteristics of both veins and arteries, types of leg veins, definition of VTE, definition of DVT, the most common site and risk site for DVT, causes, risk factors, signs \& symptoms, laboratory investigations, diagnostic test, complications, medical treatment of DVT and nursing management of patients with HS.

DOI: 10.9790/1959-0601025162 $\quad$ www.iosrjournals.org $\quad 52 \mid$ Page


Part $(\boldsymbol{C})$ : Nurses' knowledge about NCSs for preventing DVT which included 39 items about;

- General information about NCSs $\rightarrow$ (3items).

- $\quad$ Standard (1) nursing assessment $\rightarrow$ (7 items)

- Standard (2) nursing diagnosis $\rightarrow$ one item

- $\quad$ Standard (3) expected outcome $\rightarrow$ one item

- Standard (4) nursing planning $\rightarrow$ two items

- Standard (5) nursing activities implementation(24items)

- $\quad$ Standard (6) evaluation $\rightarrow$ one items

Scoring system for knowledge:

Knowledge obtained from nurses was scored and calculated. Each question was ranged from $0-1$ grade. Whereas, correct answer scored 1 grade and score zero for incorrect response. The total score level for the questionnaire sheet was 63 grades (equal 100\%).

- $\quad \geq 80 \%$ considered satisfactory knowledge $\rightarrow(\geq 50.4$ score $)$.

- $\quad<80 \%$ considered unsatisfactory knowledge $\rightarrow(0-<50.4$ score $)$.

Second tool: Nurses' practice observational checklist:

It was adapted from ${ }^{[4][9]}$ and modified by researcher to be applicable by staff nurses working in orthopedic unit at Benha university hospital. It consisted of (200) criteria (sub items) under main six headings that represented the NCSs components for preventing DVT among patients undergoing HS.As following:

1 -Nursing assessment $\rightarrow$ which included:Pre-operative assessment \& Post-operative assessment

- $\quad$ Pre-operative assessment (5 items) $\rightarrow(15$ sub items $)$

- $\quad$ Post-operative assessment (4 items) $\rightarrow(9$ sub items)

- Items related to assessing venous blood flow using Doppler device for femoral vein $\rightarrow(7$ sub items $)$ : this procedure was not applicable in both pre and post assessment due to Doppler device was not available in orthopedic unit .so, grades related to this procedure were not included in the total score.

2. Nursing diagnosis $\rightarrow$ (3 sub Items)

3. Expected outcome $\rightarrow$ ( 8 sub items $)$

4. Nursing planning $\rightarrow$ (8 sub items)

5. Nursing activities implementation $\rightarrow$ (3 main items) mechanical, pharmacological and health education measures for preventing DVT.

- Mechanical measures for preventing DVT consist of; encouraging leg elevation (4 sub items), performing postoperative range of motion exercise (31 sub items), teaching postoperative isometric exercise (16 sub items), using walker for ambulation (24 sub items), using graduated elastic compression stocking (22 sub items), and applying intermittent pneumatic compression device (IPC) (8 sub items): this procedure was not applicable due to IPC device was not available in orthopedic unit .so, grades related to this procedure were not included in the total score.

- Pharmacological measures for preventing DVT $\rightarrow(39$ sub items $)$

- Health education measures for preventing DVT $\rightarrow(7$ sub items $)$

6. Evaluation $\rightarrow(6 \mathrm{sub}$ items $)$

Scoring system:

The total scores of observational checklist 384 grades equal $100 \%$, zero was given for not done, one grade was given for done incorrect and two grades was given for done correct. The satisfactory started from $80 \%$ and above and unsatisfactory were below $80 \%$.

Third tool: Hospital structure related to preventing DVT observational checklist: It was adopted from ${ }^{[4]}$.It was included 9 items as follows: DVT preventing policy, DVT preventing guidelines, DVT preventing consultants, training sessions for nurses regarding preventing DVT among patients undergoing HS, supervision of nurses performance regarding preventing DVT, facilities and equipment for DVT assessment procedure, DVT preventing facilities and supplies including: for ambulation using walker, for intermittent pneumatic compression (IPC) device, for pharmacological prevention and computer programs for electronic alerts for patients at risk of DVT and preventive measures, health education booklet regarding preventing DVT and documentation sheet.

Scoring system: Each item was scored as follows:Zero =not availableand $(1)=$ available.

Fourth tool: Patients' assessment sheet: It included three parts:

Part (I) demographic characteristics of patients undergoing HS which included age, gender, residence, level of education, marital status and occupation.

Part (II): DVT risk assessment sheet: it was adopted from ${ }^{[10]}$ andincluded patients' medical, past, family history for DVT and smoking.

Part (III): patients' outcome consisted of clinical signs and symptoms as well as, incidence of DVT before discharge from orthopedic unit and after one month (during follow up at outpatient clinic) 


\section{Scoring system of parts (II \& III)}

Each item was scored as follows: Zero $=$ absent. $(1)=$ present.

\section{II-Operational design:}

\section{1-Preparatory phase:}

This phase included the following; reviewing the available literature and different studies related to research problem, and theoretical knowledge of its various aspects of the study, using textbooks, evidencebased articles, internet periodicals and magazines in order to collect tools of this study and NCSs for preventing DVT among patients undergoing HS. This phase took six month from the beginning of April 2014 to the end of September 2014.

\section{2- Ethical consideration:}

All ethical issues were taken into consideration during all phases of the study; the ethical research consideration in this study included the following: the research approval was obtained before standard implementation, the objectives and aim of the study were explained to all participants and the researcher maintained anonymity and confidentiality of the subjects.

\section{3-Tools validity and reliability:}

Testing validity was tested though a jury of 7 experts who composed of 1 professors, 1 assistant professor and 1 lecturer from faculty of nursing ,Zgazig University, 2 assistant professors and 1 lecturer from faculty of nursing , Benha University , 1 professor from faculty of medicine ,Benha University. The experts reviewed the tools for clarity, relevance, comprehensiveness, simplicity and applicability, minor modification were done. This phase took one month from the beginning to the end of October 2014.

Testing reliability of proposed tools was done by Cronbachx alpha test.

\section{4- Pilot study}

A Pilot study was carried out on $10 \%$ of the studied subjects, who were included in the main study. The pilot study was done to ensure clarity, applicability, feasibility of conduction of the study tools, and time needed for each tool to be filled in. Some modifications were done according to the pilot study findings. This phase took one month from the beginning to the end of November 2014.

\section{5-Field work:}

The process of data collection was carried out from the beginning of December 2014 to the end of Jun 2015. The researcher visited the orthopedic unit at Benha university hospital, three days weekly (morning \& afternoon) to collect the data by using previous tools. The researcherinterviewedwith the available nurses in orthopedic unit, introduced herself to initiate communication, explained the aim of the study and took their approval to participate in the study prior to data collection, then the researcher assessed the nurses' performance level (knowledge and practice) regarding preventing DVT among patients undergoing HS by using questionnaire sheet and observational checklist for nurses and hospital structure (pre NCSs implementation) as following:

Firstly, the researcher observed nurses' practice using the observational checklist based on the designed NCSs then the researcher filled out the observational checklist sheet to assess their practice pre implementing the NCSs. The average time needed for the completion of each observational checklist took about $25-35$ minutes.Each nurse was evaluated 3 times and the mean was calculated. In addition the researcher fulfills the checklist items related to hospital structure for preventing DVT. It took about 15 minutes.

Secondly, the questionnaire sheets were administered by the researcher to all nurses individually to assess their knowledge about NCSs for preventing DVT among patients undergoing HS. The average time needed for the completion of each interview took about 25 - 35 minutes and explanation of the questionnaire sheet was done by the researcher after an official permission to conduct this study was obtained from the head of the orthopedic units and nurses' consent. This period of pretests (knowledge and practice) took one month. To facilitate the implementation of the designed NCSs, researcher prepared the training places, teaching aids and media (pictures, handouts). This was followed by arranging for the training sessions schedule based on the contents of booklet, numbers of staff nurses involved, time availability, shifts as well as the resources available. After that for conducting the training sessions; the nurses were divided into small groups (10 groups) and each group contained 2 to 3 nurses. Each group of nurses chose the optimal time (10-11 am) for morning shift and (4-5pm) for afternoon shift whenever, they have minimal workload.

The implementation phase was achieved through training sessions at a period of 2 weeks for each group. This phase took a period of five months in addition to one month for preprogram baseline assessment and another one month for post program evaluation. Taking into consideration the use of Arabic language that suits the level of the nurses. Motivation and reinforcement during training sessions were used in order to enhance motivation for the sharing in this study. The total number of sessions for each group of the nurses included in this study was 6 sessions, 2 sessions for theoretical part and 4 sessions for practical part. The duration of session ranged between 45 minutes to 60 minutes, including 10 minutes for discussion and feedback. Each session usually started by a summary of what has been taught during the previous session and the objectives of the new topics. Feedback 
and reinforcement of teaching was performed according to the nurses' needs to ensure their understanding.The content of theoretical part included an overview about DVT, nursing management of patients with HS and NCSs for preventing DVT. The content of practical part included nursing assessment, nursing diagnosis, expected outcome, nursing planning and applying nursing intervention regarding mechanical, pharmacological and health education measures for preventing DVT. Teaching methods for theoretical part were lecture and group discussion, meanwhile for practical part were demonstration and remonstration. Media utilized were handouts, videos and data show. The content of training sessions covered in booklet. Each nurse obtained a copy of Arabic booklet.The evaluation phase was achieved after implementing the NCSs, the post tests were administered to assess nurses' knowledge and their practice using the same forms of pretest and the researcher assess patients outcome in both groups (study and control) using the patients assessment sheet before patients discharge from orthopedic unit and after one month in the outpatient orthopedic clinic. This period of posttests (knowledge and practice) took one month.

\section{Administrative Design:}

Permission was granted from the Dean of faculty of nursing, Benha University, hospital directors and head of orthopedic unit and the outpatient orthopedic clinic at Benha university hospital. Obtain the approval for data collection. The objectives and the nature of the study were explained and then it was possible to carry out the study with minimum resistance. Additional oral consent was taken from the nurses and patients who participate in the study after explanation of the nature, aims and expected outcomes of the study.

\section{Statistical Design:}

The collected data were organized, coded, computerized, tabulated and analyzed by using the statistical package for social science (SPSS), version (18). Data analysis was accomplished by the use of number, percentage distribution, mean, standard deviation, chi-square test was used to determine significant for nonparametric variable and correlation coefficient and multiple linear regression analyses was used to test the significance of some variances. A significant level value was considered when $p<0.05$ for testing the research hypothesis while, highly significant level value was considered when $\mathrm{p}<0.001$.

Limitation of the study:

1. It was difficult to collect all nurses together at the same time to attend the teaching sessions. So, the researcher obliged to divide the nurses into groups and state schedule with optimal time sessions for them. Also, sometimes the researcher had to repeat the missed session for nurses individually.

2. This study was performed on purposive small sample size in the selected sitting and depend on subjective data for detecting the incidence of DVT (patient assessment sheet)among patients undergoing HS and not using objective data (diagnostic test for example Doppler) to confirm the occurrence of DVT.

3. Doppler device and intermittent pneumatic compression device (IPC) were not available in orthopedic unit. So, the items related this procedure was not applicable.

\section{Results}

Table (1) demonstrated the distribution of nurses according to their demographic characteristics, $(50.0 \%)$ of the study subject were in the age category (> 30 years old) with a mean age of $(31.3667 \pm 8.70784$ years), the majority of them $(93.3 \%)$ were female and married $(90 \%)$. According to level of education, nursing diploma (secondary school) was the highest proportion (76.7\%). More than half of them $(56.7 \%)$ had an experience more than 10 years. and (100\%) of them were not attending any previous training courses about NCSs for preventing DVT.

Figure (1) revealed that distribution of studied nurses according to their total knowledge scores level pre and post NCSs implementation, (30\%) of the studied nurses were having a satisfactory knowledge level pre NCSs implementation. However, the majority of nurses $(96.7 \%)$ were having a satisfactory knowledge level post its implementation. Withhighly statistically significant differences observed as paired t test $8.694(\mathrm{P} \leq 0.001)$.

Figure (2) revealed that, (20\%) of the studied nurses had satisfactory level of practice pre NCSs implementation. However, post its implementation, the majority of nurses $(93.3 \%)$ had satisfactory level of practice. Withhighly statistically significant differences observed as paired t test $14.116(\mathrm{P} \leq 0.001)$.

Table (2) revealed that, there were a highly statistically significant positive correlation between nurses' total knowledge and their total practice score pre \&post NCSs implementation observed as $(r=0.582 \& r=0.695$ with $P$ value $<0.001)$ respectively.

Table (3)showed that, more than one third of the studied patients in both study and control groups were in age category more than 60 years old $(36.4 \& 45.4 \%)$, more than half of them were males $(54.5 \% \& 68.2 \%)$, the highest portion of them were in rural area $(59.1 \% \& 71.2 \%),(45.4 \% \& 30.3 \%)$ were illiterate and more than two third of them were married $(75.8 \%$ and $77.3 \%)$ respectively and with no statistically significant differences $\mathrm{P}>0.05$. 
Table (4): showed that, distribution of hospital structure items for preventing DVT, all items regarding hospital structure were not available, meanwhile, facilities and equipment for DVT assessment procedure ascentimeter tape, lukewarm gel and base line coagulation laboratory investigations and DVT preventing facilities and supplies as walker and anticoagulant drugs were available (100\%) respectively.

Table (5) showed thatdistribution of clinical signs and symptoms of DVT among studied patients in both study and control groups, there were decrease in all items related to clinical signs and symptoms of DVT in study group than in control group during and after one month from discharge and with statistically significant differences observed as $(\mathrm{P} \leq 0.001 \& \mathrm{P}<0.05)$.

Figure (3) indicated that, the incidence rate of DVT among patients undergoing HS in study group was lesser than patients in control group with percentage of $(4.5 \& 13.6 \%)$ respectively and with highly statistically significant differences observed as $(\mathrm{P} \leq 0.001)$.

Table (1): Distributionof the studied nurses according to their demographic characteristics $(\mathrm{N}=30)$

\begin{tabular}{|c|c|c|}
\hline Demographic characteristics & \multicolumn{2}{|c|}{$\mathbf{N}=\mathbf{3 0}$} \\
\hline Nurses age & No. & $\%$ \\
\hline $19<25$ & 3 & 10.0 \\
\hline $25-30$ & 12 & 40.0 \\
\hline$>30$ & 15 & 50.0 \\
\hline Mean \pm SD & \multicolumn{2}{|c|}{$31.3667 \pm 8.70784$} \\
\hline \multicolumn{3}{|l|}{ Gender } \\
\hline Male & 2 & 6.7 \\
\hline Female & 28 & 93.3 \\
\hline \multicolumn{3}{|l|}{ Marital status } \\
\hline Single & 2 & 6.7 \\
\hline Married & 27 & 90.0 \\
\hline Widow & 1 & 3.3 \\
\hline \multicolumn{3}{|l|}{ Level of education } \\
\hline Diploma (secondary school) & 23 & 76.7 \\
\hline Diploma + specialty & 0 & 0.0 \\
\hline Technical nursing institute & 4 & 13.3 \\
\hline Bachelor degree & 3 & 10.0 \\
\hline \multicolumn{3}{|l|}{ Yearsof experience } \\
\hline$<1$ year & 3 & 10.0 \\
\hline $1<5$ year & 3 & 10.0 \\
\hline 5-10year & 7 & 23.3 \\
\hline$>10$ year & 17 & 56.7 \\
\hline Mean \pm SD & \multicolumn{2}{|c|}{$12.1333 \pm 6.31218$} \\
\hline \multicolumn{3}{|l|}{ Attendance of training courses } \\
\hline No & 30 & 100.0 \\
\hline
\end{tabular}

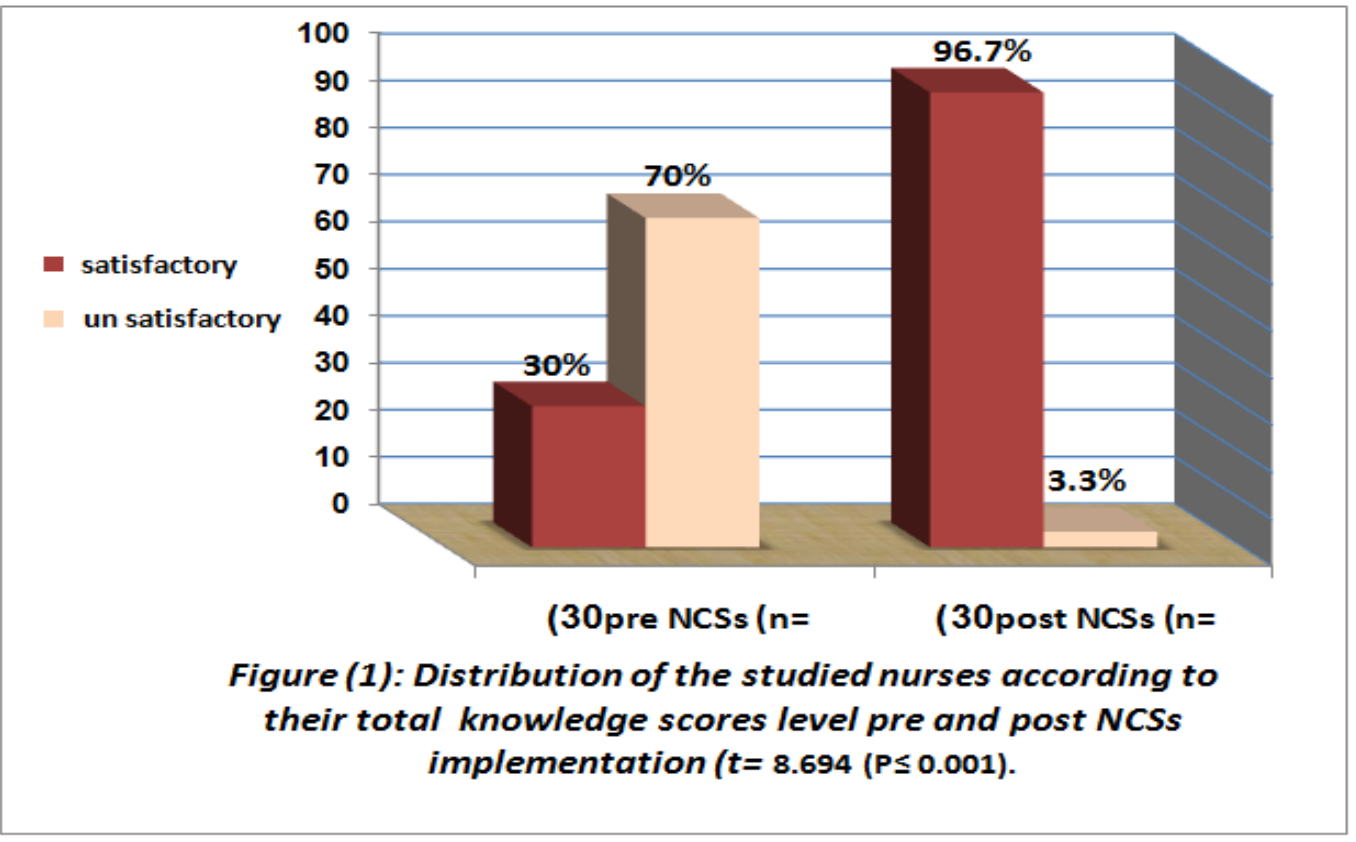




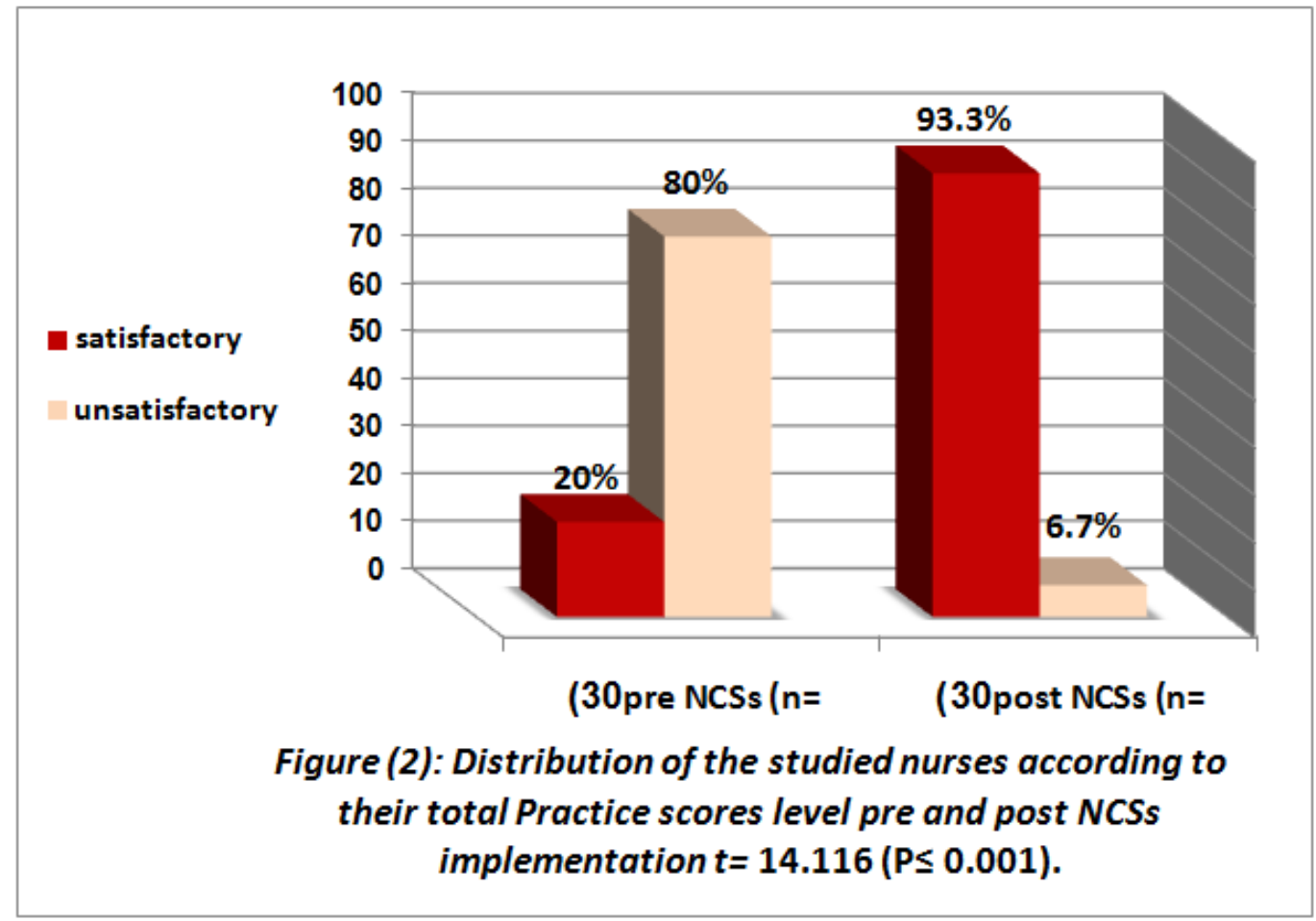

Table (2): Correlation coefficient between total nurses' knowledge and practice score pre and post NCSs implementation

\begin{tabular}{|l|l|l|}
\hline \multirow{2}{*}{ Nurses' knowledge } & \multicolumn{2}{c|}{ Nurses' practice } \\
\cline { 2 - 3 } & \multicolumn{1}{|c|}{ Pre NCSs implementation } & Post NCSs implementation \\
\hline $\mathrm{r}$ & 0.582 & 0.695 \\
\hline $\mathrm{P}$ value & $<0.001^{* *}$ & $<0.001 * *$ \\
\hline
\end{tabular}

Table (3): Distribution of the studied patients demographic characteristics in both study and control groups

\begin{tabular}{|c|c|c|c|c|c|c|}
\hline \multirow{2}{*}{$\begin{array}{c}\text { Demographic characteristics of } \\
\text { the patients }\end{array}$} & \multicolumn{2}{|c|}{$\begin{array}{c}\text { Control group } \\
n=66\end{array}$} & \multicolumn{2}{|c|}{$\begin{array}{c}\text { Study group } \\
n=66\end{array}$} & \multirow[t]{2}{*}{$\chi^{2}$} & \multirow[t]{2}{*}{$P$ value } \\
\hline & No & $\%$ & No & $\%$ & & \\
\hline \multicolumn{7}{|l|}{ Age: } \\
\hline$<20$ year & 6 & 9.1 & 3 & 4.5 & \multirow{5}{*}{$\begin{array}{l}14.96 \\
0\end{array}$} & \multirow{5}{*}{$>0.05$} \\
\hline $20<40$ year & 11 & 16.7 & 12 & 18.2 & & \\
\hline $40-60$ year & 19 & 28.8 & 27 & 40.9 & & \\
\hline$>60$ year & 30 & 45.4 & 24 & 36.4 & & \\
\hline Mean \pm SD & \multicolumn{2}{|c|}{$62.455 \pm 10.292$} & \multicolumn{2}{|c|}{$61.576 \pm 8.8249$} & & \\
\hline \multicolumn{7}{|l|}{ Gender: } \\
\hline Male & 45 & 68.2 & 36 & 54.5 & \multirow[t]{2}{*}{2.747} & \multirow[t]{2}{*}{$>0.05$} \\
\hline Female & 21 & 31.8 & 30 & 45.5 & & \\
\hline \multicolumn{7}{|l|}{ Residence: } \\
\hline Rural & 47 & 71.2 & 39 & 59.1 & \multirow[t]{2}{*}{3.184} & \multirow[t]{2}{*}{$>0.05$} \\
\hline Urban & 19 & 28.8 & 27 & 40.9 & & \\
\hline \multicolumn{7}{|l|}{ Educational level: } \\
\hline Illiterate & 20 & 30.3 & 30 & 45.4 & \multirow{6}{*}{$\begin{array}{l}10.96 \\
0\end{array}$} & \multirow{6}{*}{$>0.05$} \\
\hline Read and write & 16 & 24.2 & 10 & 15.2 & & \\
\hline Prep school & 6 & 9.1 & 0 & 0.00 & & \\
\hline Secondary school & 14 & 21.2 & 20 & 30.3 & & \\
\hline Technical institute & 5 & 7.6 & 4 & 6.1 & & \\
\hline University & 5 & 7.6 & 2 & 3.0 & & \\
\hline \multicolumn{7}{|l|}{ Marital status: } \\
\hline Single & 7 & 10.6 & 8 & 12.1 & \multirow[b]{2}{*}{ 7..075 } & \multirow[b]{2}{*}{$>0.05$} \\
\hline Married & 51 & 77.3 & 50 & 75.8 & & \\
\hline
\end{tabular}




\begin{tabular}{|c|c|c|c|c|c|c|}
\hline Widow & 6 & 9.1 & 8 & 12.1 & & \\
\hline Divorced & 2 & 3.0 & 0 & 0.00 & & \\
\hline \multicolumn{7}{|l|}{ Occupation: } \\
\hline Not working & 16 & 24.2 & 18 & 27.3 & \multirow{5}{*}{0.570} & \multirow{5}{*}{$>0.05$} \\
\hline Worker & 17 & 25.8 & 8 & 12.1 & & \\
\hline Housekeeping & 15 & 22.7 & 20 & 30.3 & & \\
\hline Student & 7 & 10.6 & 3 & 4.5 & & \\
\hline Employee & 11 & 16.7 & 17 & 25.8 & & \\
\hline
\end{tabular}

Table (4): Distribution of hospital structure items for preventing DVT among patients undergoing HS

\begin{tabular}{|c|c|c|c|c|}
\hline \multirow{2}{*}{ Hospital structure } & \multicolumn{2}{|c|}{ available } & \multicolumn{2}{|c|}{ Not available } \\
\hline & No & $\%$ & No & $\%$ \\
\hline DVT preventing Policy & 0 & 0.00 & 30 & 100.0 \\
\hline DVT preventing guidelines & 0 & 0.00 & 30 & 100.0 \\
\hline DVT preventing consultants & 0 & 0.00 & 30 & 100.0 \\
\hline $\begin{array}{l}\text { Training sessions for nurses regarding preventing } \\
\text { DVT among patients undergoing hip surgery }\end{array}$ & 0 & 0.00 & 30 & 100.0 \\
\hline $\begin{array}{l}\text { Supervision of nurses performance regarding } \\
\text { preventing DVT }\end{array}$ & 0 & 0.00 & 30 & 100.0 \\
\hline \multicolumn{5}{|l|}{$\begin{array}{l}\text { Facilities and equipment for DVT assessment } \\
\text { procedure }\end{array}$} \\
\hline Centimeter tape & 30 & 100.0 & 0 & 0.00 \\
\hline Doppler device & 0 & 0.000 & 30 & 100.0 \\
\hline Lukewarm gel & 30 & 100.0 & 0 & 0.00 \\
\hline $\begin{array}{l}\text { Base line coagulation laboratory investigations } \\
\text { (PT, PA, INR) }\end{array}$ & 30 & 100.0 & 0 & 0.00 \\
\hline \multicolumn{5}{|l|}{ DVT preventing facilities and supplies } \\
\hline For ambulation using walker & 30 & 100.0 & 0 & 0.00 \\
\hline $\begin{array}{l}\text { For Intermittent pneumatic compression (IPC) } \\
\text { device }\end{array}$ & 0 & 0.000 & 30 & 100.0 \\
\hline $\begin{array}{l}\text { Pharmacological measures preventing DVT ( } \\
\text { anticoagulant drugs) }\end{array}$ & 30 & 100.0 & 0 & 0.0 \\
\hline $\begin{array}{l}\text { Computer programs for electronic alerts for } \\
\text { patients at risk of DVT and preventive } \\
\text { measures. }\end{array}$ & 0 & 0.00 & 30 & 100.0 \\
\hline Health education booklet for preventing DVT & 0 & 0.00 & 30 & 100.0 \\
\hline Documentation sheet & 0 & 0.00 & 30 & 100.0 \\
\hline
\end{tabular}

Table (5): Distribution of studied patients in both study and control groups according to patient's outcome

\begin{tabular}{|c|c|c|c|c|c|c|c|c|c|c|}
\hline \multirow{3}{*}{$\begin{array}{l}\text { Patient's outcome(clinical } \\
\text { signs and symptoms DVT) }\end{array}$} & \multicolumn{4}{|c|}{$\begin{array}{l}\text { Control group } \\
n=66\end{array}$} & \multicolumn{4}{|c|}{$\begin{array}{l}\text { Study group } \\
\mathrm{n}=66\end{array}$} & \multirow{3}{*}{$\begin{array}{l}\text { Chi- } \\
\text { square } \\
\chi^{2}\end{array}$} & \multirow[t]{3}{*}{$P$ value } \\
\hline & \multicolumn{2}{|c|}{$\begin{array}{l}\text { During } \\
\text { discharge }\end{array}$} & \multicolumn{2}{|c|}{$\begin{array}{l}\text { After one } \\
\text { month }\end{array}$} & \multicolumn{2}{|c|}{$\begin{array}{l}\text { During } \\
\text { discharge }\end{array}$} & \multicolumn{2}{|c|}{$\begin{array}{l}\text { After one } \\
\text { month }\end{array}$} & & \\
\hline & No & $\%$ & No & $\%$ & No & $\%$ & No & $\%$ & & \\
\hline \multicolumn{11}{|c|}{ Leg pain or tenderness in one or both leg } \\
\hline Present & 25 & 37.9 & 29 & 43.9 & 10 & 15.2 & 3 & 4.5 & \multirow[t]{2}{*}{14.636} & \multirow[t]{2}{*}{$<0.001 * *$} \\
\hline Absent & 41 & 62.1 & 37 & 56.1 & 56 & 84.8 & 63 & 95.5 & & \\
\hline \multicolumn{11}{|l|}{ Swelling in one or both legs } \\
\hline Present & 14 & 21.2 & 16 & 24.2 & 7 & 10.6 & 5 & 7.6 & \multirow[t]{2}{*}{10.473} & \multirow[t]{2}{*}{$<0.001 * *$} \\
\hline Absent & 52 & 78.8 & 50 & 75.8 & 59 & 89.4 & 61 & 92.4 & & \\
\hline \multicolumn{11}{|c|}{ Warmth in the skin of the affected leg } \\
\hline Present & 19 & 28.8 & 10 & 15.2 & 8 & 12.1 & 3 & 4.5 & \multirow[t]{2}{*}{15.760} & \multirow[t]{2}{*}{$<0.001 * *$} \\
\hline Absent & 47 & 71.2 & 56 & 84.8 & 58 & 87.9 & 63 & 95.5 & & \\
\hline \multicolumn{11}{|c|}{ Red or discolored skin in the affected leg } \\
\hline Present & 17 & 25.8 & 9 & 13.6 & 11 & 16.7 & 8 & 12.1 & \multirow[t]{2}{*}{7.876} & \multirow[t]{2}{*}{$<0.05 *$} \\
\hline Absent & 49 & 74.2 & 57 & 86.4 & 55 & 83.3 & 58 & 87.9 & & \\
\hline \multicolumn{11}{|l|}{ Visible veins surface } \\
\hline Present & 15 & 22.7 & 5 & 7.6 & 14 & 21.2 & 1 & 1.5 & \multirow[t]{2}{*}{7.526} & \multirow[t]{2}{*}{$<0.05^{*}$} \\
\hline Absent & 51 & 77.3 & 61 & 92.4 & 52 & 78.8 & 65 & 98.5 & & \\
\hline
\end{tabular}




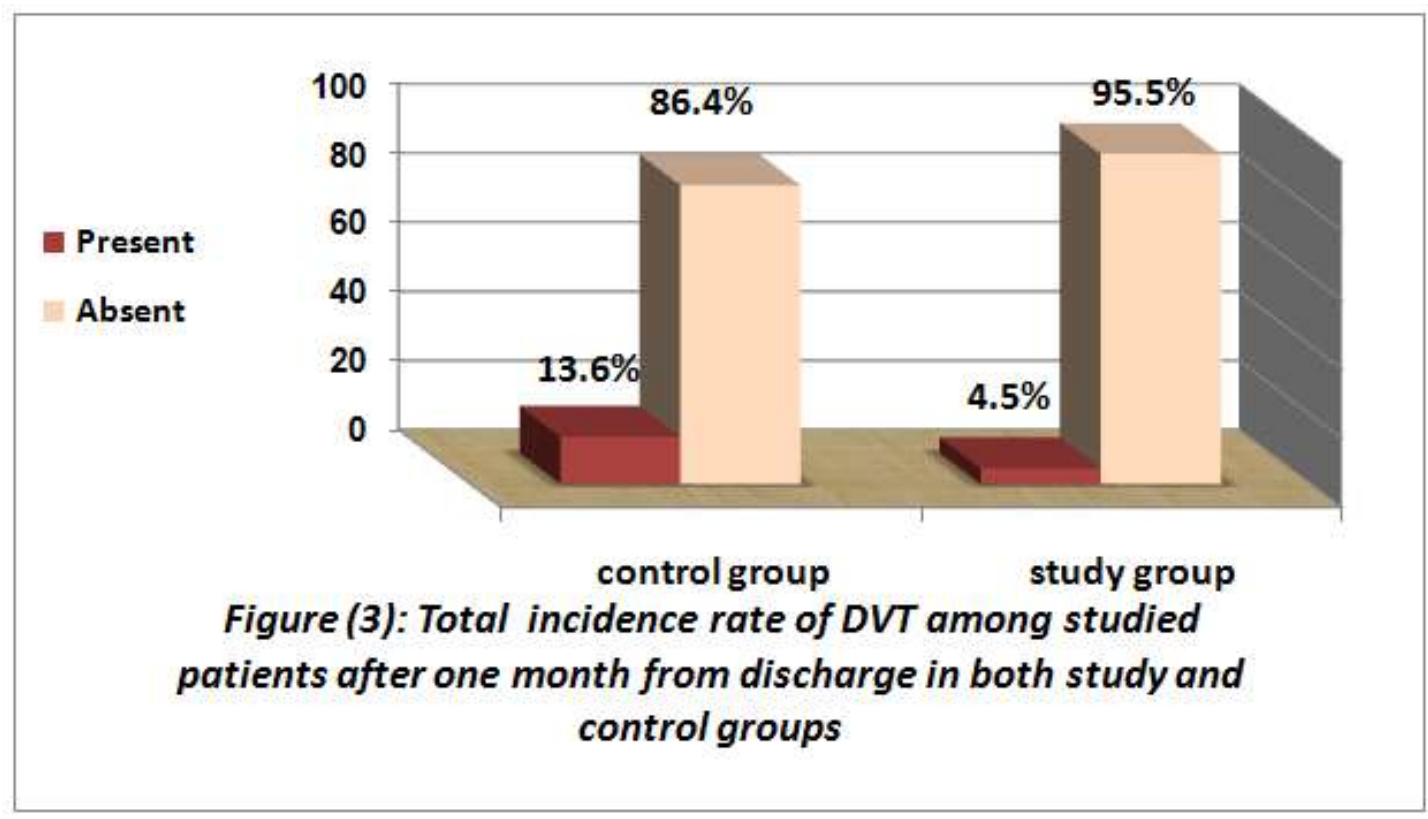

\section{Discussion}

The aim of the present study was to evaluate the effect of nursing care standards for preventing deep vein thrombosis among patients undergoing hip surgery on nurses' performance and patients' outcome at Benha University Hospital.Regarding age, the present study revealed that about half of the studied subjects were in the age category $>30$ years old. This might be due to most of nurses were diploma level of education and prefer to work in general medical surgical unit not in critical department. This result agreed with the study conducted by $^{[11]}$ about "effectiveness of the planned teaching program on DVT among the staff nurses of selected hospital" and found that, most of nurses in age category $>30$ years old. This result disagreed with the study conducted in orthopedic unit at Alexandria university hospital by ${ }^{[12]}$ about" knowledge and performance among nurses before and after a training program on patient falls" and found that the highest percentage of nurses aged between 18 years to less than 25 years accounted for $55 \%$ of the total sample.

As regard to nurses' gender, the present study revealed that the majority of nurses were females. This might be due to overall ratio of male nurses to the female nurses were less in the nursing profession. As regard to marital status of the studied nurses, the finding of the present study revealed that the highest percentage of them was married. According to the level of education for nurses the finding of the present study indicated that the majority of nurses had diploma in nursing education. This might be due to the newly nurses with bachelor degree in nursing education were distributed in critical care unit rather than other units in the hospital. This finding disagreed with the study conducted by ${ }^{[13]}$ about "efficacy of implementing nursing care protocol on total hip replacement patient's outcome in orthopedic department at Tanta university hospital" and founded that the majority of studied nurses were Baccalaureate degree in nursing education.

Concerning to years of experiences for nurses the finding of the present study revealed that more than half of them had an experience more than 10 years. This might be due to stability of the most nurses in their places from their appointed in the orthopedic unit.As regarding attendance of nurses training courses, the present study showed that all nurses had not attending any previous training courses about nursing care standards for preventing DVT. This finding is supported by ${ }^{[12][13]}$ who documented that the majority of the studied nurses hadn't attended any previous training program.

As regarding total nurses' knowledge scores level about NCSs for preventing DVT pre and post NCSs implementation. The current study revealed that about one third of the studied nurses were having a satisfactory knowledge level pre NCSs implantation. However, the majority of nurses were having a satisfactory knowledge level post its implementation. The researcher thinks that the reasons of these results might be due to lack of educational and training background for nurses under the study whereas, almost all the staff nurses did not receive formal training courses about NCSs for preventing DVT among patients undergoing HS. In addition, the majority of the nurses had only diploma in nursing education in which the content of NCSs for preventing DVT was limited in their curriculum. This result is in congruent with ${ }^{[11]}$ who documented the majority of staff nurses had unsatisfactory level of knowledge pre implementation planned teaching program on DVT than post implementation. 
The study result is in the same line with the study conducted by ${ }^{[14]}$ about "knowledge, attitude and practices of health care providers towards DVT prophylaxis in five teaching hospitals of Rawalpindi" and reported that the knowledge of health care providers about DVT prophylaxis were less than adequate. As well as these findings agreed with the study conducted by ${ }^{[15]}$ about "evaluation of hospital nurses perceived knowledge and practices of VTE assessment and prevention" and indicated that there were lack of nurses' knowledge and recommended that a substantial need for focused education about VTE prevention for hospital nurses and support for hospital to monitor VTE care.

As regarding total nurses' practice scores level about NCSs for preventing DVT pre and post NCSs implementation.The findings of the present study revealed that less than quarter of the studied nurses had satisfactory level of practice pre NCSs implementation. However, post its implementation, the majority of nurses had satisfactory level of practice.The researcher thinks that this result might be due to the majority of DVT preventing hospital structures items were not available. In this respect ${ }^{[16]}$ who emphasized that lack of hospital supportive system that includes all hospital structure standards which included lack of DVT prophylaxis policy and guidelines, lack of training for health care providers and inadequate facilities for efficient implementation of DVT prophylaxis measures were barriers that led to poor performance of health care providers for implementing DVT prophylaxis. Another significant reason for total unsatisfactory level of nurses practice might be due to the largest proportion of their level of knowledge had unsatisfactory pre NCSs implementation.

This finding was supported by ${ }^{[17]}$ who stressed that variability in nurses' knowledge of DVT assessment and appropriate prevention appeared to be a negative influence on implementation strategies. Also, this finding agreed with ${ }^{[12]}$ who founded that knowledge and practices of healthcare providers about DVT prophylaxis in hospitalized patients were less than ideal and added that hospitals need to develop their own guidelines for preventing DVT.

Overall, the results of the current study indicated that nurses weren't provided satisfactory nursing practice regarding preventing DVT for patients undergoing HS pre NCSs implementation. This might be due to the majority of nurses didn't have enough knowledge about NCSs as well as lack of written NCSs for preventing DVT, lack of in-service training programs, lack of orientation programs for newly employees and lack of a system for supervision and evaluation of nursing practice. The previous interpretations were in the same line with ${ }^{[18]}$ who stated that extending nurses' knowledge is very much needed to improve nurses' practice and to prepare them for their extending roles and hospital requirement. Also, added that NCSs were important to promote, guide, and direct professional nursing practice, important for self-assessment and evaluation of practicing nurses and recommended that today's practicing nurse must be aware of nursing standards.

In relation between total nurses' knowledge and their practice score, the current study documented that there were a highly statistically significant positive correlation pre and post NCSs implementation. This finding indicated that practice can be easily improved especially if linked with relevant scientific base of knowledge. This finding agreed with ${ }^{[11]}$ who found that there were a highly statistically significant relation and positive correlation between total nurses` knowledge and their total practice score pre and post implementing DVT teaching program. Also, this finding agreed with ${ }^{[19]}$ who documented that there were highly statistically significant relation between nurses' knowledge and their practice post NCSs implementation.

The finding of the current study reported that nurses who had unsatisfactory levels of knowledge and/or inadequate practice about DVT preventing standard pre the NCSs implementation with statistically significant improvement post NCSs implementation. This finding is in the same line with ${ }^{[20]}$ who documented that nurses who had poor levels of knowledge and/or skills before the exposure to a training program had a significant improvement after the implementation of the program. In this respected ${ }^{[21]}$ documented that the training program has beneficial effect in improving the nurses` knowledge and skills. They also recommended that educational programs should be organized according to the needs of nurses with continuous evaluation and adopting proper checklists for work monitoring to enhance patient and staff awareness; lead to reduced process errors, mitigating overall risks, eventually resulting in effective patient care.

Concerning to hospital structure for preventing DVT .The finding of the present study revealed thatpolicy structure, guideline structure, training session structure, supervision of nurses' performance regarding preventing DVT structure, IPC device supplies structure for preventing DVT,computer programs for electronic alerts for patients at risk of DVTdocumentation sheet for DVT assessment, application of DVT preventive measures and HS patient education were not available in hospital structure. These findingsare incongruent with ${ }^{[22]}$ who stressed that hospital policies on thromboprophylaxisare an essential first step as they enable seniorclinicians to consider the evidence. Also the current result disagreed with ${ }^{[23]}$ who stated thatguidelines that recommend for every healthcare facility should have a written policy on continuous quality improvement related to DVT risk assessment, diagnosis and prevention. And this result disagreed ${ }^{[24]}$ stressed that educational and training sessions for nurses regarding preventing DVT were considered starting points of any quality improvement initiative that help in decreasing the incidence of DVT. 
In relation to DVT preventing facilities and supplies structure regarding walker for ambulation and pharmacological preventions, the present study indicated that these facilities and supplies were available in hospital structure and these considered an important items in hospital structure standard in the established DVT preventing standard.As regarding age and gender of the studied patients in both study and control groups, the finding of the current study indicated that more than one third of them were in age category more than 60 years old and more than half of them were males. The researcher thinks that this result might be due to this age group considered high risk factors for exposure to hip fracture and the needed for surgical repair by hip surgery procedure. This finding in the same line with ${ }^{[25]}$ who found that hip fracture occurs at any age but $90 \%$ of hip fractures occur in people older than age 65 years. As regarding the level of education of the studied patients in both study and control groups, the finding of the present study documented that about one third of them were illiterate. This finding agreed with ${ }^{[26]}$ who found that the majority of studied patients in both study and control groups were illiterate.

As regarding theincidenceand clinical signs \& symptoms of DVT among studied patients in both study and control groups, the present study revealed that the incidence of DVT among patients undergoing HS in study group lesser than patients in control group and with highly statistically significant differences. The researcher thinks that this result might be due to improvement in nurses' performance for preventing DVT among studied patients post NCSs implementation which had a positive effect on study group. This finding agreed with ${ }^{[27]}$ who noticed that after intervention the patients of studied group had an improvement of DVT clinical assessment as evident by absences of warm leg and tenderness in the leg than patients of control group who follow the routine care only. Moreover the study conducted by ${ }^{[28]}$ about "reduction of DVT incidence in intensive care after a clinician education program" and documented that one year ICU based educational programs on implementation of DVT prophylaxis were associated with a significant decrease in DVT incidence and also in the length of stay in ICU.

Finally, it can be concluded that the developing NCSs for preventing DVT among patients undergoing HS achieved objectives by improving nurses knowledge and their practice and application of these care by nursing staff help in reducing and/or preventing DVT for these patients. Also there was reducing the incidence and signs \& symptom of DVT among study group post implementation.

\section{Conclusion}

In the light of the study findings, it might be concluded that, the majority of studied nurses had unsatisfactory performance level (knowledge and practice) regarding NCSs for preventing DVT among patients undergoing HS pre NCSs implementation. While, orthopedic nurses' performance level for prevention DVT were statistically significantly improved post NCSs implementation.Also, the study findings showed that implementation of the developed NCSs for preventing DVT were statistically significantly effective in improving patients' outcome including reducing incidence of DVT among patients with HS in study group than patients in control group.

\section{Recommendation}

In the light of the results of the current study, the following recommendations are suggested:

- Orientation programs should be provided for all newly nurses about the developed NCSs for preventing DVT among patients undergoing HS.

- Orthopedic hospital administrative system should provide DVT preventing policy and guidelines for raising nurses' awareness about DVT as a serious problem, availability of DVT preventing NCSs as evidence for best practice and the importance of collaborative work between physicians and nurses in order to decrease incidence of developing DVT among patients.

- Adequate facilities supplies and equipment that enhance the implementation of DVT preventing standards including Doppler and intermittent pneumaticcompression devices and documentation records.

- Further studies should be conducted on a larger sample obtained from different geographical areas in Egyptto evaluate the effect of NCSs implementation for preventing DVT in the incidence of DVT occurrence among patients undergoing HS using subjective data (patient assessment sheet) and objective data (diagnostic test for example Doppler).

- Health education booklet for preventing DVT should be available and distributed for all patients with HS.

\section{References}

[1]. Korucu, I.H., Turkmen, F., Kacira, B., Bilge, O., Kilicaslan, A. and Toker, S. (2014):Thromboprophylaxis for Hip and Knee Arthroplasty: Current Managements and Review of the Literature. World Journal of Cardiovascular Diseases, 4: 586 593.Located@www.scirp.org/journal/PaperDownload.aspx?paperID: Accessed at 14 December 2015.

[2]. Lee,S.Y., Hyun Ro, D., Chung, C.Y., Lee, K.M., Kwon, S.S., Sung, K.H. and Moon Seok Park, M.S. (2015): Incidence of Deep Vein Thrombosis after Major Lower Limb Orthopedic Surgery: Analysis of a Nationwide Claim Registry. Yonsei Med J; 56(1): 139-145. Accessed at 20 February 2015 
[3]. Garcia, F.L., Marins, M.H.T., Raddi, T.B. and Picado, C.H.F. (2015): Unfractionated heparin and mechanical thromboprophylaxis in hip arthroplasty. ActaOrtop Bras; 23(4):208-11. Available from URL: http://www.scielo.br/aob: Accessed at October 21, 2015.

[4]. Al Tawil1, F.A., Abou-Donia, S.A. and Abdel Gawad, M. (2013): Establishing Nursing Standards for Prophylaxis of Deep Vein Thrombosis among Patients Undergoing Hip Surgery. Journal of American Science, 9 (1):406420.Located@www.jofamericanscience.org/.../061_14040am0901_40: Accessed at October 25, 2015.

[5]. Khoon Ho, W (2010): Deep vein thrombosis Risks and diagnosis. Australian Family Physician Vol. 39, (7); pp. 468- 473

[6]. Anthony, M. (2013): Instructions for continuing nursing education contact hours: nursing assessment of deep vein thrombosis. CNE Med Surg Nursing; 22(2): 95-98.

[7]. American Nurse Associations, (ANA) (2010): standards of professional nursing practice. Nursing: Scope and Standards of Practice, $2^{\text {nd }}$ Edition. Silver Spring, MD: Nursesbooks.org:3-46 located @ https://www.iupuc.edu/.../nursing/.../Website\%20-\%20A. Accessed at 16-December 2015.

[8]. Collins, R., MacLellan, L., Gibbs, H., MacLellan, D. and Fletcher, J. (2008): Venous thromboembolism prophylaxis: The role of the nurse in changing practice and saving lives. Ustralian Journal of Advanced Nursing; 27 (3): 84- 88

[9]. Mike, W., Anderson, L. and Kelly, H. (2012): Venous thromboembolism (VTE) prevention and management policy and procedures, North Cumbria University hospitals NHS Trust: 2-39. Located at www.ncuh.nhs.uk/about-us/...of.../00153503.pdf.Accessed at October 15, 2015.

[10]. National Council of State Boards of Nursing (NCSBN), (2009): The effect of high fidelity simulation on prelicensure nursing student performance and knowledge: A Pilot Study presented at the NCSBN midyear meeting, 2(3):54

[11]. Das, S., Sahoo, A. and swain, R. (2014): Effectiveness of the Planned Teaching Program on Deep Vein Thrombosis among the Staff Nurses of selected hospital. IOSR Journal of Nursing and Health Science (IOSR-JNHS), 3(1):48-51. Located @iosrjournals.org/iosr-jnhs/papers/vol3.../I03134851.pdf: Accessed at October 15, 2015.

[12]. Abou El Enein, N.Y., Abd El Ghany, A. S. and Zaghloul, A. A. (2012): Knowledge and performance among nurses before and after a training programme on patient falls. Open journal of Nursing; 2 (1):358-364. Locatedhttps://www.google.com.eg/file.scirp.org/pdf/OJN20120400004_13043015.pdf : Accessed at October 21, 2015.

[13]. El Shemey, M.B.A and Elsaay, E.A.E (2015): Efficacy of implementing nursing care protocol on total hip replacement patient's outcome in orthopedic department at Tanta university hospital. IOSR Journal of Nursing and Health Science (IOSR-JNHS); 4(5): 118-132. Located athttps://www.google.com.eg/iosrjournals.org/iosrjnhs/papers/...T0453118132.pdf.Accessible at Jun 24, 2016

[14]. Bhatti, A. M., Ahsin, S., Salim, B. and Mansoor, J. (2012): knowledge, attitude and practices of health care providers towards deep vein thrombosis prophylaxis in five teaching hospitals of Rawalpindi. J Ayub Med Coll Abbottabad, 24 (2): 136-149. Located @ www.ncbi.nlm.nih.gov/pubmed/24397075: accessed at16-11-2015.

[15]. Lee, J., Grochow, D., Darke, D., Johnson, L. and Reed, P. (2014): Evaluation of hospital nurses' perceived knowledge and practices of venous thromboembolism assessment and prevention. Journal of Vascular Nursing; 32 (1): 18-24.

[16]. Worel, J. (2009): venous Thromboembolism: what is preventing achievement of performance measures and consensus? J CardiovascNurs.Nov-Dec; 24(6): S9-14.

[17]. Bratzler, D.W. (2010): Development of National Performance Measures on the Prevention and Treatment of Venous Thromboembolism. J Thromb Thrombolysis Feb; 29 (2): 148-154: Accessed at November 2, 2015.

[18]. McMahon, D. (2011): Nursing standards of practice, HG experts.com. Located at http://www.hg experts. Com/article. asp?id= 6237: Accessed at October 12, 2015.

[19]. Ghanem, H.M.,and El-khayat, R.A., (2012): "chronic subdural hematoma: Effect of developing and implementing postoperative nursing care standards on nurses performance for reduction or prevention postoperative complication, Journal of American Science, 8 (2),p.694, located @ www.jofamericanscience.org/.../096 8394am0802 686: Accessed at November 15, 2015.

[20]. Sayed, S.Y., Ghanem, H.M., Mohamed, W.Y. and El-Gamal, T.A. (2011): Micro vascular free tissue transfer surgeries: Impact of a designed teaching protocol on nurse's performance for reduction or prevention of post-operative flap failure. Life Science journal; 8(2): 158-170. Located @ www.lifesciencesite.com/.../life0802/20_4808life0802 : Accessed at 2 December 2015.

[21]. Ookalkar, D.S. (2009): Effect of training program on nurses' performance. International Journal of Quality and Reliability Management; 26 (8): 817- 830.

[22]. Amin, A.N. and Deitelzweig, S.B. (2009): Optimizing the prevention of venous thromboembolism: recent quality initiatives and strategies to drive improvement. Comm J Qual Patient Saf; 35(11):558-64.

[23]. Guyatt, G.H., Akl, E.A., Crowther, M. and Gutterman, D.D. (2012): Antithrombotic therapy and prevention of thrombosis American College of Chest Physicians Evidence Based Clinical Practice Guideline. Chest journal, 9 ${ }^{\text {th }}$ Edition; 141(2): S7-S47. Located at journal.publications.chestnet.org/issue.aspx?issueid... Accessed at March22, 2015.

[24]. Michota, F.A. (2007): Bridging the gap between evidence and practice in venous thromboembolism prophylaxis: the quality improvement process. Gen Intern Med. Dec; 22(12):1762-70.

[25]. Bakhshi, H, Alavi-Moghaddam, M, Imam, M, and Banasiri, M (2012): D-Dimer as an Applicable Test for Detection of Posttraumatic Deep Vein Thrombosis in Lower Limb Fracture. The American Journal of Orthopedics® (AJO); vol 41(6); pp 78.

[26]. El Shemey, M.B.A and Elsaay, E.A.E (2015): Efficacy of implementing nursing care protocol on total hip replacement patient's outcome in orthopedic department at Tanta university hospital. IOSR Journal of Nursing and Health Science (IOSR-JNHS); 4(5): 118-132. Located athttps://www.google.com.eg/iosrjournals.org/iosrjnhs/papers/../T0453118132.pdf.Accessible at Jun 24, 2016.

[27]. Gad, S.S. and El-Sheikh, A.A. (2013): Effect of Mechanical Measures on Prevention of Deep Vein Thrombosis among Genera Surgical Patients. Med. J. Cairo Univ., 81 (1):1043-1051. Located @ www.medicaljournalofcairouniversity.net : accessed at 4 November 2015

[28]. Baddi, M. (2010): Reduction of deep vein thrombosis incidence in intensive care after a clinician education program. Journal of Thrombosis and Hemostasis; 8(1):121-128. 